\title{
Critical Lens in Learning Analytics Research: A Systematic Literature Review
}

\author{
Marcia Cristina Moraes', James E. Folkestad ${ }^{1}$, Daniel Birmingham ${ }^{1}$ \\ ${ }^{1}$ School of Education - Colorado State University (CSU) \\ Fort Collins - CO - USA \\ \{marcia.moraes, james.folkestad, daniel.birmingham\}@colostate.edu
}

\begin{abstract}
This paper describes a systematic literature review that aims to answer the following research question: How has critical theory been used in learning analytics research? Nine previous literature review and fifteen studies were analyzed. Results showed that none of the previous literature reviews considered critical theory as a component of their analyzes and the topics of algorithm bias, ethics, justice and prediction are among the most studied on works that used Critical lens in Learning Analytics. Considerations are drawn to support future works that promotes the use of Critical lens in Learning Analytics and in the design of adaptive systems for learning and teaching.
\end{abstract}

\section{Introduction}

Learning analytics (LA) emerged as an independent area from the field of academic analytics in 2010, and papers related to LA draw on a diverse range of literature from fields such as education, technology and social sciences [Ferguson, 2012]. This diversity reflects in the works that have been done, such as predicting students' success [Gasevic et al., 2016], detecting learning strategies [Gasevic et al., 2017], designing learning analytics [Shibani et al. 2019], learning analytics visualizations [McKenna et al. 2019], and students' vulnerability [Prisloo and Slade, 2016].

Acknowledging that data does not speak for itself and that the selection and use of student data are not neutral acts but embedded in social, political, economic and cultural agendas [Selwyn, 2014] and considering that several works in LA are being done in the fields of education and social science, this paper aims to answer the following research question: How has critical theory been used in learning analytics research?

In order to answer that question, we conducted a systematic literature review as defined by Petticrew and Roberts (2006) as a method of making sense of a large bodies of information, mapping out areas of uncertainty, and identifying where little or not relevant research has been done, but where new studies are needed. As a result, we have the purpose of providing evidence-based information to support and develop practices involving the use of critical theory in LA and applying it to adaptive systems for learning and teaching.

\section{Background}

In this paper we are using the definition of learning analytics adopted by the Society for Learning Analytics Research (SoLAR) [Ferguson, 2012, p.3]: "Learning analytics is the 
VIII Congresso Brasileiro de Informática na Educação (CBIE 2019)

Anais do XXX Simpósio Brasileiro de Informática na Educação (SBIE 2019)

measurement, collection, analysis and reporting of data about learners and their contexts, for purposes of understanding and optimizing learning and the environments in which it occurs". This definition encompasses two beliefs: that LA makes uses of preexisting data and that LA techniques can help to analyze with those data.

Several assumptions can be made on how to handle data, considering the lenses that researchers have about the nature of knowledge (epistemology), how knowledge is acquired and accumulated (methodology), and to what end we apply such knowledge (teleology). In this paper we use the Critical lens and consider that the values entered into the inquiry and the choice of a particular value systems tends to empower certain persons while disempowering others and as such values certain knowledge over others (epistemology), it is necessary to bring "true consciousness" of their conditions to the disempowered (oppressed) (methodology) to drive the transformation of their realities (teleology) [Lincoln et al., 2011]. We assume that in social science, critical theory "references systematic though attempting an explicit analysis toward social justice, which distinguished it from typical mainstream theory" [Anyon, 2008, p. 2]. We believe that Critical lens should be considered in LA works to give voice to the oppressed and help them to transform their realities. In this way, we used these definitions and conceptual framework to drive our systematic literature review.

\section{Methodology}

According to Petticrew and Roberts (2006) the first step to be done in a systematic literature review is to find existing systematic reviews in the area. To do that we conducted a search considering the sources presented in table 1. These sources were selected because they are known for publishing works related to education, social science and computer science areas. We consider papers and book chapters written in English from the last five years (2015-2019) because we would like to have an overview of the most recent works done in the area. The keywords used were "learning analytics and literature review." We found nine previous literature reviews, but none of them is related to critical theory aspects. The results from this search will be presented in section 4.1.

Table 1. Sources of the bibliographical research

\begin{tabular}{|l|l|}
\hline Databases & $\begin{array}{l}\text { Google Scholars, ACM Digital Library, Willey Online } \\
\text { Library, IEEE Library }\end{array}$ \\
\hline Journals & $\begin{array}{l}\text { Computers \& Education, Computers in Human Behavior, } \\
\text { and Journal of Learning Analytics }\end{array}$ \\
\hline Proceedings & Learning Analytics and Knowledge Conference \\
\hline
\end{tabular}

The second step was to search for papers that were published in LA and have relation with critical theory. We used the following keywords for our automatic search: "critical theory and learning analytics", "equity and learning analytics", "social justice and learning analytics", "inclusion and learning analytics", "power and learning analytics", "intersectionality and learning analytics", "praxis and learning analytics", "privilege and learning analytics", "vulnerability and learning analytics", and "minority and learning analytics", customized to the specific syntax for each database. We selected these keywords because these terms represent major constructs used and/or investigated in critical theory. Again, we only look for papers and book chapters published in English from the last five years (2015-2019), to have a picture of the most recent research. In 
some cases, the search resulted in a large number of results; the number of total distinct results is not reported because searching different bases and using the term "learning analytics" within all keywords returned many repeated results across all searches. For the searches carried on Google Scholars we took into consideration only the first 25 results, for the other databases and the Computer \& Education and Computers in Human Behavior journals we considered the first 100 results (because after that the titles did not have any relationship with the critical keywords used in the search), and since the Journal of Learning Analytics does not have a search engine, we read all titles and abstracts of published papers since 2015. Searches were performed on April and May.

After the first stage of automatic search using keywords, we filtered out less relevant literature based on the titles and abstracts, using the Critical lens described in the background section, and, if necessary, skimming through the text. We also included a search in the reference section for each selected paper in order to find additional relevant papers (snowball technique). At the end of this process, we ended up with 15 works that used Critical lens, 14 are papers and 1 is a book chapter. The book chapter was published in 2017. Table 2 presents those works by the year of publication. As we can observe, the number of publications is low, but it is increasing since 2016. The analysis of these literature will be presented in section 4.2.

Table 2. Number of publications by year

\begin{tabular}{|c|c|}
\hline Year of Publication & Number of Publications Considered \\
\hline 2015 & - \\
\hline 2016 & 2 \\
\hline 2017 & 3 \\
\hline 2018 & 5 \\
\hline 2019 & 5 \\
\hline
\end{tabular}

\section{Results}

\subsection{Previous Literature Review on Learning Analytics}

This review was done as a first step in our methodology for a systematic literature review process, and it revealed that previous works did not review LA in the perspective of Critical lens. After analyzing the nine previous literature reviews, we observe that five addressed benefits and challenges in LA, four of them related to general benefits and challenges and one specifically related to LA policies in Higher Education (HE); two addressed LA aspects for enhance learning; one addressed visual learning analytics; and one deep learning applied to LA.

The works that investigated the benefits and challenges in LA in general were Avella et al. (2016), Viberg et al. (2018), Banihashem et al. (2018), Alfy et al. (2019); and related to LA policies was Tsai and Gasevic (2017). Avella et al. (2016) revealed that several works on LA used data mining to predict, cluster and discover student models and emphasized the separation of data from human judgment to analyze data, and that these benefited course offerings, curriculum development and students learning outcomes. Some of the challenges identified were the lack of connection to learning science and ethical and privacy issues. Viberg et al. (2018) found that since 2016 most of the work in LA was starting to move from prediction about grades and retention to a deeper 
VIII Congresso Brasileiro de Informática na Educação (CBIE 2019)

Anais do XXX Simpósio Brasileiro de Informática na Educação (SBIE 2019)

understanding of students' learning experiences, however more than $80 \%$ of the works reviewed did not approach ethical issues (such data privacy and informed consent) in a systematic way. Banihashem et al. (2018) findings showed that one of the most important challenges in LA in education was ethics and privacy along with the lack of attention to theoretical foundations. As benefits, the authors pointed out that LA could increase students engagement, improve learning outcomes, identify academically at-risk students, and provide real-time feedback and personalization of learning. Some of the benefits presented by Alfy et al. (2019) were monitoring and predicting students' performance and helping academically at-risk students to succeed. Among the challenges identified was concerns about ethical use of data and data security. Tsai and Gasevic (2017) recognized six challenges to the adoption of LA: shortage of leadership to ensure that implementation of LA is strategically planned and monitored, shortage of equal engagement of different stakeholders, shortage of pedagogical-based approach, insufficient training opportunities to give users the ability to employ learning analytics, shortage of empirical studies to validate the impact of LA, and limited availability of LA policies that address issues of privacy and ethics as well as the previous challenges listed.

$\mathrm{Si} \mathrm{Na}$ and Tasir (2017) conducted a review on how LA interventions contributes to student success. The interventions had purposes of engagement, retention, performance outcome, motivation and collaborative learning and stated that LA interventions helped at-risk students. Mangoroska and Giannakos (2018) reviewed how analytics is being used to drive design to enhance learning. They found that the use of LA for learning design is related to discover learning phenomena as the moment of learning or misconception, and that design improved and pedagogically sound learning environments.

The paper on visual learning analytics [Vieira et al., 2018] reviewed the works considering two perspectives: the approaches designers and researchers have used to visualize educational data, and how the fields of information visualization and education has been used in the design of visual LA tools. Their findings suggest that few visualizations consider background information from students, such as prior performance and demographics; little work has been done to use LA tools visualization in classroom; the most common kind of visualizations are traditional statistical visualizations such as bar plots and scatter plots; and there is a lack of studies that employ sophisticated visualizations and engage deeply with educational theories.

The review on deep learning (DL) applied to LA [Coelho and Silveira, 2017] found that publications using DL started to appear in 2015 and that were three times more papers using Artificial Neural Network than DL during the period of the review. The main educational task reported were: computer assisted instruction, student performance prediction, students' assessment, student modelling, and intelligent tutoring systems.

\subsection{Use of Critical Lens in LA}

After analyzing the 15 studies using Discourse Textual Analysis (DTA) [Moraes and Galiazzi, 2007], nine topics emerged from the studies and some have sub-topics (table 3).

Table 3. Studies categorized by topics and sub-topics

\begin{tabular}{|l|l|}
\hline \multicolumn{1}{|c|}{ Topic } & \multicolumn{1}{|c|}{ Studies } \\
\hline Algorithm Bias & $\begin{array}{l}|c| \\
\text { 2019] [Lang, 2019] }\end{array}$ \\
\hline
\end{tabular}


VIII Congresso Brasileiro de Informática na Educação (CBIE 2019)

Anais do XXX Simpósio Brasileiro de Informática na Educação (SBIE 2019)

\begin{tabular}{|c|c|}
\hline Justice & \\
\hline Structural & [Johnson, 2017] \\
\hline Social & [Aguilar, 2018a] \\
\hline At-Risk Students & [Aguilar, 2018b] \\
\hline Students Vulnerability & [Prisloo and Slade, 2016] \\
\hline Intersectionality & [McKay et al., 2018] \\
\hline Datafication & [Jones and McCoy, 2018] \\
\hline Ethics & \\
\hline Principles & [West et al., 2016] \\
\hline in Justice and Care & [Prinsloo and Slade, 2017] \\
\hline In Praxis & [Jones and McCoy, 2019] \\
\hline Prediction using & \\
\hline Social Economics & [Rizvi et al. 2019] \\
\hline Culture and Gender & [Benson and Filippaios, 2019] \\
\hline
\end{tabular}

Regarding the algorithm bias topic, Knox (2017) considered that culture is not only embedded in learning analytics systems but is also reshaped through the power of its algorithms. In this sense, the author developed a project that considered student participation and choice in the learning analytics process as an attempt to foster critical awareness of data capture and analysis. Leavy (2018) study revealed that the over representation of man in the design of algorithms for machine learning could undo decades of advances in gender equality and that gender balance is crucial to prevent algorithms from perpetuating gender ideologies that disadvantage women. The author proposed a computational approach to identify gender bias that could be used to remove it from training data for machine learning algorithms. Meaney and Fikes (2019) identify two kinds of bias that can influence learning analytics, early-adopter iteration bias that may lead LA to derive insights about optimal course design considering preferences and behaviors patterns of more prepared students, not considering students that need more attention from instructors (at-risk students), and research-bias praxis that compounds the potential problems from early-adopter iteration bias in two separate but interrelated ways. First, practitioner used the skewed data from early-adopter iteration bias produced by LA researchers. Second, little research has been produced to examine how particular mindsets and processes of practitioners that produce Virtual Learning Environments (VLEs) may impact the design of those environments and students' outcomes. Lang (2019) analyzed how penalties for assessment purposes are perceived by different groups of risk-tolerant students. The author suggested that penalties should not be used for assessment purposes (embedding penalties for random guessing) because this could influence populations that are underserved groups (females, underrepresented minorities, and low socioeconomic status) to not interact with the platform and replicate existing inequalities.

The topic of justice was treated in different ways by the literature examined. Johnson (2017) mentioned that privacy, individuality, autonomy and algorithm discrimination are the major ethical concerns in LA and based on these concerns, the author proposed a framework of structural justice that focused on how informational, operational and organizational structures of LA influence students' abilities of selfdevelopment and self-determination. Aguilar (2018a) acknowledge that LA has the potential do contribute to a more equitable and socially just educational outcomes for students who are at risk of being neglected. This is possible according to the author 
VIII Congresso Brasileiro de Informática na Educação (CBIE 2019)

Anais do XXX Simpósio Brasileiro de Informática na Educação (SBIE 2019)

because LA techniques can bring personalized instruction, reducing the classroom size to 1. Doing so, it is possible to consider the particularities of each student and bringing social justice to education at least on an individual level.

Aguilar (2018b) studied how self-data visualization is related to at-risk students' motivations. The author considered at-risk students that: are first generation, who come from low socioeconomic backgrounds, who attended low-performing high schools, who attended large urban schools, who attended small rural schools, and who are underrepresented in the academy. Results suggested that visualizations that contains selffocused affordances evoked statements centered on mastering materials, while visualizations containing comparative information (information about students and class average), evoked responses that disheartened students and/or made them feel accountable to do better.

Prisloo and Slade (2016) proposed a framework for learner agency that aimed to decrease student vulnerability, increase their agency, and empower them as participants in learning analytics. The framework stated that institutions need to made clear what data is collected, for what purposes and with whom the data may be shared and under what conditions. Learners could opt to have their data collected and shared or not.

The topic intersectionality was examined by McKay et al. (2018) considering how the feminist studies of intersectionality have informed the analysis of how social identity might influence student performance in large introductory science courses. The authors applied intercategorial complexity to analyze gender (male, female) and ethnicity; and intracategorical complexity to examine a specific gender considering anomalies in performance (males underperforming and female performing better than expected).

Jones and McCoy (2018) argued that data should be considered a kind of document and approaches informed by documentation studies will enable to understand how students are constructed into data considering the social and political shaping of data in LA. Authors mentioned that more attention needs to be given to this.

West et al. (2016) argued that ethical principles should underpin institutional decision making in relation to LA. Authors proposed and ethical decision-making framework that encourages institutional leaders and people involved in implementing LA to consistently apply and document ethical decision-making processes to develop a wellaligned and transparent institutional policies that considers ethical literacy. Prinsloo and Slade (2017) stated that analysis and application of student data are not neutral acts and all flow from and perpetuate social, political, economic and cultural agendas. Considering that the work in HE should be a moral practice that embodies ethics of care, the authors proposed a framework that considers the ethics of justice and the ethics of care. Jones and McCoy (2019) presented a method from science and technology studies (STS), social-technical integration research of STIR that situates social scientists alongside learning analytics practitioners to engage the latter in questions about ethics in praxis and social consequences of their work. One proposed way to address ethics in praxis is to use participatory/co-design strategy to include stakeholders in the algorithm creation resolving ethical problems before they are implemented in LA technologies.

Rizvi et al. (2019) used socio-economic strata to predict the risk of failure in online learning. Authors found that region, neighborhood poverty level, and prior 
VIII Congresso Brasileiro de Informática na Educação (CBIE 2019)

Anais do XXX Simpósio Brasileiro de Informática na Educação (SBIE 2019)

education are strong predictors of overall learning outcomes. Benson and Filippaios (2019) used culture (nationality) and gender as variables to predict user engagement in social networking sited.

\section{Discussion and Conclusion}

As we can observe from the description of the previous literature review (section 4.1), a lot of work has been done regarding prediction models. Although the focus has been moved to approaches that considers students' learning experiences, there are still few works relating LA to educational theories and addressing ethical issues of use of data. Moreover, privacy and data use are the most considered aspect in ethics that has been examined. None of the previous systematic literature overview analyzed and approached ethics considering the critical lens and looked for topics such as ethics of justice, ethics of care, and ethics of epistemology, for example. This gap in the literature review drove us to acquire a general picture of the evidence of how critical theory has been used in LA in order to direct future research efforts.

After conducting a systematic literature review as proposed by Petticrew and Roberts (2006) and following the methodology presented in section 3, we analyzed 15 works that addressed critical lens concepts. With this evidence-based information we present some considerations and ideas to contribute to practice involving the use of critical theory in LA. Although our study has limitations, as it only considered English works from the last five years, we believe that we gave a first step to bring more light for such an important aspect of LA and hope to raise discussions among the community.

Few of these studies made explicitly clear which critical theory they used as a philosophical framework to guide their work. It seems that it is not only a deep relation with theories of learning that LA papers are missing, as mentioned by Viera et al. (2018), but also a deep relation with education paradigms as well. Future studies should make it explicitly both the learning theory as well as the philosophical framework that support and guide them. By doing that, researchers should state what types of knowledge are valued or silenced in their studies, as well as how they are contributing to bring "true consciousness" [Freire, 1972] to the oppressed and leading to the transformation of their realities. Topics such as ethics of: justice, care, and epistemology should be considered.

Algorithm bias was the most studied topic in the works reviewed, followed by ethics, justice, and prediction. All these works mentioned in some level how LA could help bring equality for students, but none of them mentioned how to bring equitability in LA. Considering that equality and equitability are not synonymous and that equality connotes sameness in treatment and equity "is associated with fairness or justice in the provision of education or other benefits and it takes individual circumstances into consideration" [Espinoza, 2007, p. 345], future works with Critical lens should go beyond equality, aiming to reach equitability.

Intersectionality, that is looking how different aspects of humans (gender, race, and class, for example) interact and intersect in individual's access to social, political and economic institutions (Crenshaw, 1991), should be considered in LA studies that aims to educational equity and social justice. This should be a component to be considered for researchers that works in personalization as a kind of social justice in LA, as well as in the different kinds of prediction studies that are conducted in the field and can be used in 
VIII Congresso Brasileiro de Informática na Educação (CBIE 2019)

Anais do XXX Simpósio Brasileiro de Informática na Educação (SBIE 2019)

adaptive systems for learning and teaching. One possible way to start working on that is using demographic data available in academic systems to know students that are part of a minority group and start building models to understand how the variables present in those group affect, and impact predictions results and how to use those variables in the design of adaptive systems for learning and teaching. Researchers could also conduct qualitative ethnographic studies with students of those identified minority groups to understand their needs. Those understandings should be shared with instructors and university stakeholders in order to take actions that help minorities to succeed in their academic lives.

\section{References}

Aguilar, S. J. (2018a). Learning Analytics: at the Nexus of Big Data, Digital Innovation, and Social Justice in Education. Tech Trends, 62, 37-45.

Aguilar, S. J. (2018b). Examining the Relationship Between Comparative and SelfFocused Academic Data Visualizations in At-Risk Students' Academic Motivation. Journal of Research on Technology in Education, 50(1), 84-103.

Alfy, S. E., Gómez, J. M., Dani, A. (2019). Exploring the benefits and challenges of learning analytics in higher education institutions: a systematic literature review. Information Discovery and Delivery, 47(1), 25-34.

Anyon, J. (2008). "Introduction: Critical Social Theory, Educational Research, and Intellectual Agency". In J. Anyon (Ed.), Theory and Educational Research, p. 124. New York: Routledge.

Avella, J. T., Kebritchi, M., Nunn, S. G., Kanai, T. (2016). Learning Analytics Methods, Benefits and Challenges in Higher Education: A Systematic Literature Review. Online Learning, 20(2), 13-29.

Banihashem, S. K., Aliabadi, K., Ardakani, S. P., Delaver, A., Ahmadabadi, M. N. (2018). Learning Analytics: A Systematic Literature Review. Interdisciplinary Journal of Virtual Learning in Medical Sciences, 9(2), 1-10.

Benson, V., Filippaios, F. (2019). The role of learning analytics in networking for business and leisure: A study of culture and gender differences in social platform users. Computers in Human Behavior, 92, 613-624.

Coelho, O. B., Silveira, I. F. (2017). "Deep Learning applied to Learning Analytics and Educational Data Mining: A Systematic Literature Review”. In XXVIII Simpósio Brasileiro de Informática na Educação (SBIE 2017), p. 143-152.

Crenshaw, K. (1991). Mapping the Margins: Intersectionality, Identity Politics, and Violence against Women of Color. Stanford Law Review, 43(6), pages 1241-1299.

Espinoza, O. (2007). Solving the equity-equality conceptual dilemma: a new model for analysis of the educational process. Educational Research, 49(4), 343-363. 
VIII Congresso Brasileiro de Informática na Educação (CBIE 2019)

Anais do XXX Simpósio Brasileiro de Informática na Educação (SBIE 2019)

Ferguson R. (2012). Learning analytics: drivers, developments and challenges. Int J Tech Enhanced Learn (IJTEL), 4(5/6), 304-317.

Freire, P. (1972). Pedagogy of the oppressed. New York: Herder and Herder.

Gasevic, D., Jovanovic, J., Pardo, A., \& Dawson, S. (2017). Detecting learning strategies with analytics: Links with self-reported measures and academic performance. Journal of Learning Analytics, 4(2), 113-128.

Gasevic, D., Dawson, S., Rogers, R. and Gasevic, D. (2016). Learning analytics should not promote one size fits all: The effects of instructional conditions in predicting academic success. In The Internet and Higher Education, 68-84. Elsevier.

Johnson, J. A. (2017). Ethics and Justice in Learning Analytics. New Directions in Higher Education, 179, 77 - 87.

Jones, K. M., McCoy, C. (2018). Reconsidering data in learning analytics: Opportunities for critical research. Learning, Media and Technology, 1-19.

Jones, K. M., McCoy, C. (2019). "Ethics in Praxis: Social-Technical Integration Research in Learning Analytics". In Companion Proceeding of the $9^{\text {th }}$ International Conference on Learning Analytics \& Knowledge (LAK'19), Fairness and Equity in Learning Analytics Systems Workshop, p. 5-14.

Knox, J. (2017). Data Power in Education: Exploring Critical Awareness with the "Learning Analytics Report Card". Television and New Media, 18(8), 743-752.

Lang, D. (2019). "Strategic Omission and Risk Aversion: A Bias-Reliability Tradeoff". In Companion Proceeding of the $9^{\text {th }}$ International Conference on Learning Analytics \& Knowledge (LAK'19), Fairness and Equity in Learning Analytics Systems Workshop, p. 20-28.

Leavy, S. (2018). "Gender Bias in Artificial Intelligence: The Need for Diversity and Gender Theory in Machine Learning". In $1^{\text {st }}$ International Workshop on Gender Equality in Software Engineering, p. 14-16.

Lincoln, Y. S., Lynham, S. A., Guba, E. G. (2011). Paradigmatic controversies, contradictions, and emerging confluences, revisited. In Denzin, N. K., Lincoln, Y. S. (Eds.), The SAGE handbook of qualitative research, p. 97-128. LA: SAGE.

Mangoroska, K., Giannakos, M. (2018). Learning analytics for learning design: A systematic literature review of analytics-driven design to enhance learning. IEEE Transactions of Learning Technologies (Early Access), 1-19.

McKay, T. A., Grom, G., Koester, B. (2018). Categorization, Intersectionality, and Learning Analytics. In Companion Proceeding of the $8^{\text {th }}$ International Conference on Learning Analytics, p. 1-5.

McKenna, K., Folkestad, J. E., Moraes, M. C. (2019). "Reflections of Visual Form Learning Analytics: Spaced Retrieval Practice Activity". In Companion 
VIII Congresso Brasileiro de Informática na Educação (CBIE 2019)

Anais do XXX Simpósio Brasileiro de Informática na Educação (SBIE 2019)

Proceeding of the $9^{\text {th }}$ International Conference on Learning Analytics \& Knowledge (LAK'19), Practitioner Track, p. 20-25.

Meaney, M., Fikes, T. (2019) "Early-adopter Iteration Bias and Research-praxis Bias in the Learning Analytics Ecosystem". In Companion Proceeding of the $9^{\text {th }}$ International Conference on Learning Analytics \& Knowledge (LAK'19), Fairness and Equity in Learning Analytics Systems Workshop, p. 14-20.

Moraes, R., \& Galiazzi, M. C. (2007). Análise Textual Discursiva. Ijuí: E. Unijuí.

Petticrew, M., \& Roberts, H. (2006). Systematic Reviews in the Social Sciences: a practical guide. Oxford: Blackwell.

Prinsloo, P., \& Slade, S. (2016). Student vulnerability, agency, and learning analytics: An exploration. Journal of Learning Analytics, 3(1), 159-182.

Prinsloo, P., Slade, S. (2017). Big Data, Higher Education and Learning Analytics, Beyond Justice and Ethics of Care. B. Kei Daniel (ed.), Big Data and Learning Analytics in Higher Education, p. 109-124.

Rizvi, S., Rienties, B., Khoja, S. A. (2019). A role of demographics in online learning; A decision tree based approach. Computers \& Education, 137, 32-47.

Selwyn, N. (2014). Distrusting educational technology. Critical questions for changing times. New York: Routledge.

Shibani, A., Knight, S., and Shum, S. B. (2019). "Contextualizable Learning Analytics Design: A Generic Model, and Writing Analytics Evaluations". In Proceeding of the 9th International Conference on Learning Analytics \& Knowledge (LAK'19), p. $210-219$.

Si Na, K., Tasir, Z. (2017). “A Systematic Review of Learning Analytics Intervention Contributing to Student Success in Online Learning". In 2017 International Conference on Learning and Teaching in Computing and Engineering, p. 62-68.

Tsai,Y., Gasevic, D. (2017). "Learning Analytics in Higher Education - Challenges and Policies: A Review of Eight Learning Analytics Policies". In Proceedings of the $7^{\text {th }}$ International Conference on Learning Analytics \& Knowledge (LAK'17), p. 233-242.

Viberg, O., Hatakka, M., Bälter, O., Mavroudi, A. (2018). The current landscape of learning analytics in higher education. Computers in Human Behavior, 89, 98110.

Vieira, C., Parsons, P., Byrd, V. (2018). Visual learning analytics of educational data: A systematic literature review and research agenda. Computers \& Education, 122, 119-135.

West. D., Huijser, H., Heath, D. (2016). Putting an ethical lens on learning analytics. Education Tech Research Dev, 64, 903-922. 